\title{
Reacciones Adversas a Medicamentos en el Hospital de Suba de Bogotá
}

\author{
Sonia M. Moscoso-Veloza ${ }^{1}$, Gloria F. Ramírez-Cubillos ${ }^{2}$, José J. López- Gutierrez ${ }^{3}$ y \\ Bárbara E. Gerena-Useche ${ }^{4}$ \\ ${ }^{1}$ Químico Farmacéutico. Ropsohn Therapeutics. \\ E-mail:smmoscosov@unal.edu.co,soniamaggy@yahoo.es \\ ${ }^{2}$ Químico Farmacéutico. Laboratorios Ropsohn. email: gframirezc@unal.edu.co. \\ ${ }^{3}$ Químico Farmacéutico. M. Sc. Farmacología. Universidad Nacional de Colombia. Departamento de \\ Farmacia. E-mail: jjlopezg@unal.edu.co \\ ${ }^{4}$ Químico Farmacéutico. Hospital de Suba Segundo Nivel. E-mail: bgerena@tutopia.com
}

Recibido 5 Abril 2006/Enviado para Modificación 11 Junio 2006/Aceptado 10 de Junio 2006

\section{RESUMEN}

Objetivos Identificar, documentar y analizar las sospechas de reacciones adversas que son reportadas al programa de fármacovigilancia del Hospital de Suba, de segundo nivel de complejidad.

Metodología Estudio observacional descriptivo. Se realizó un seguimiento a todos los pacientes que acudieron a los servicios de Urgencias y Consulta Externa, en el Hospital de Suba segundo nivel de la ciudad de Bogotá por un periodo de tres meses.

Resultados Se recibieron 46 reportes de sospecha de reacciones adversas a medicamentos (RAM) de los cuales 26 (56,6 \%) correspondieron a motivo de consulta, los 20 restantes se produjeron intrahospitalariamente. El sistema gastrointestinal presentó el mayor porcentaje de reportes $(30,4 \%)$, seguido del cardiovascular (17,4\%), sistema nervioso central (13\%) y la piel (13\%), Los Grupos farmacológicos sospechosos fueron los antipsicóti$\cos (30,4 \%)$, suplementos nutricionales $(17,4 \%)$, y antiinfecciosos $(10,9$ $\%)$. La incidencia de RAM como motivo de consulta fue de 3 RAM/10 000 consultas/mes.

Conclusiones Se identificaron las gestantes, niños, adolescentes y adultos jóvenes como principales grupos de riesgo para presentar RAM. Un bajo porcentaje fue clasificado como serias y al menos la mitad se estableció que su causalidad era posible de acuerdo al algoritmo de la Organización Mundial de la Salud (OMS).

Palabras Clave: Farmacoepidemiología, utilización de medicamentos (fuente: DeCS, BIREME).

\section{ABSTRACT}

Adverse drug reactions in a Suba hospital of Bogotá 
Objective This study was carried out in order to identify, document and assess suspect of Adverse Drug Reactions (ADR) that are report to pharmacovigilance program of Suba Hospital.

Methodology It was carried out an observational, descriptive and longitudinal study, in all the patients that consulted to the services of Urgencies and External Consultation, in the Hospital of Suba of the Bogotá city.

Results During the period of study 46 reports of suspicion of ADR were received, from those which 26 (56,6\%) corresponded to consultation reason, the 20 remaining it was indoor patient. In the classification for Organ-System, the gastrointestinal system presents the highest percentage of reports $(30,4 \%)$, followed by the cardiovascular $(17,4 \%)$, nervous central system (13\%), Skin (13\%), Obstetric (10,9\%), renal system (4,3\%). The therapeutic Groups with more reported percentages of suspicion of ADR were Antipsychotic (30,4\%), Nutritional Supplements (17,4 \%), Anti-infective $(10,9 \%)$, the other pharmacological groups present similar or inferior percentages to those mentioned previously. The incidence of ADR as consultation reason was 3 ADR consultation reason/10 000 consultations / month.

Conclusions It was identify to pregnant, children and young people as a risk group to development an ADR. A low proportion it was clasificated as a serious and less than half as a possible according World Health Organization algoritm.

Key Words: Pharmacoepidemiology, drug utilization (source: MeSH, NLM).

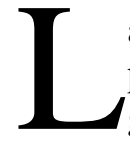

as reacciones adversas a medicamentos (RAM) se constituyen en un problema de salud pública dado que son una importante causa de ingreso hospitalario, en muchos casos aumentando los días de hospitalización y en otros constituyéndose en parte representativa de los gastos en salud (1). Dentro de los grupos de medicamentos que se han relacionado con ingresos hospitalarios se encuentran los analgésicos no esteroidales (AINES), los antitrombóticos, los corticosteroides sistémicos, los inhibidores de la enzima convertidora de angiotensina (IECA), los diuréticos, los digitálicos, los antagonistas de canales de calcio, los betabloqueadores, los nitratos, la insulina y los antidiabéticos orales (2).

La Organización Mundial de la Salud (OMS) en su preocupación por intervenir en la prevención de RAMs, ha desarrollado lineamientos para el desarrollo e implantación de programas de fármacovigilancia (FV) con el fin de establecer el perfil de seguridad de los fármacos a través de la detección de señales y optimizar el manejo de los medicamentos mediante la detección de problemas relacionados con el uso (2). 
En Colombia se han realizado algunos estudios preliminares que tienen como meta motivar la cultura del reporte voluntario y el establecimiento de programas de FV. Uno de ellos presenta una revisión de la literatura disponible, cuya finalidad es sensibilizar a los diferentes actores del sector salud sobre la necesidad de apoyar la implementación y el desarrollo de un sistema nacional de fármacovigilancia (3). Otro estudio realizó un seguimiento de los pacientes del servicio de medicina interna durante un período de cinco meses para detectar RAM. La información se recolectó mediante un formulario basado en el formato de reporte del Instituto de Vigilancia de Medicamentos y Alimentos (INVIMA), utilizando el algoritmo de Naranjo para establecer causalidad (4). Los anteriores estudios emplearon metodologías diferentes a las del presente estudio, razón por la cual no se pueden comparar los resultados obtenidos.

Adicionalmente, se han realizado esfuerzos por fortalecer las actividades de FV a través de un programa integral y estructurado adelantado por el INVIMA, el cual se enfoca en la identificación de riesgos relacionados con grupos poblacionales o grupos de fármacos, la valoración y la gestión del riesgo proponiendo intervenciones regulatorias y educativas tendientes a mejorar el uso de los medicamentos.

Con el fin de explorar las consecuencias en salud que origina el consumo de medicamentos y adicionalmente apoyar la promoción de la actividad antes mencionada, se propuso el presente trabajo, realizado en un hospital de segundo nivel de la ciudad de Bogotá. La investigación se adelantó en el Centro de Atención Medica Inmediata (CAMI) de la Localidad de Suba (Bogotá), el cual presenta un alto número de consultas en urgencias y consulta externa que facilitan la probabilidad de presentación de EA, PRM o RAM.

\section{METODOLOGÍA}

Estudio observacional descriptivo de corte longitudinal por un periodo de tiempo de tres meses. Se incluyeron todos los pacientes que acudieron a los servicios de consulta externa y urgencias del Hospital de Suba, considerado de segundo nivel de complejidad en atención, según el Sistema General de Seguridad Social en Salud de Colombia. La información fue recolectada en el Formato de Reacciones Adversas a Medicamentos (FORAM) del INVIMA y digitada en una hoja electrónica de Microsoft Excel ${ }^{\circledR}$ para su procesamiento. Para las variables cuantitativas continuas se calculó el promedio y la desviación estándar y para las variables cualitativas se estableció la proporción expresada como porcentaje. La relación causal se estableció me- 
diante el algoritmo y las categorías de causalidad de la OMS. El registro de los eventos adversos se realizó utilizando el diccionario WHOART (World Health Organization Adverse Reaction Terminology).

Se registraron todos los Eventos Adversos (EA), Problemas Relacionados con Medicamentos (PRM) o RAM (5), identificados por médicos vinculados al CAMI. El farmacéutico diligenciaba el formato del reporte completándolo con la información de la historia clínica y/o el paciente, de ser posible. Se considero una RAM como cualquier desenlace no esperado que se presenta tras la administración de un medicamento a dosis normalmente usadas para la prevención, tratamiento o alivio de enfermedades en el hombre. El EA corresponde a la misma definición, solo que en este caso no se ha establecido la relación de causalidad. El término PRM se adoptó del consenso de Granada (6) en que lo definen como resultados clínicos negativos, derivados de la farmacoterapia que, producidos por diversas causas, conducen a la no consecución del objetivo terapéutico o a la aparición de eventos no deseados.

Fueron realizadas charlas de información a los médicos y se entregó un documento orientador de diagnósticos trazadores de RAM. A los pacientes en la sala de espera se les indagó sobre la presencia de problemas de salud que hubieran presentado durante el consumo de sus medicamentos y se sugería su reporte al médico. También se dialogó con grupos de gestantes e hipertensos acerca del uso adecuado de medicamentos y algunas indicaciones generales para la adherencia a sus tratamientos.

\section{RESULTADOS}

\section{Estadísticas de consultas}

El promedio de consultas fue de 12 734/mes (desviación estándar=1 689), 8 406 consultas más que en los meses de julio y agosto, correspondiente al periodo de vacaciones escolares. Figura 1.

Distribución de los reportes

Se recibieron 46 reportes de sospechas de RAMs durante el período de estudio. De ellos 26 (56,5 \%) fueron motivo de consulta, los 20 reportes restantes se presentaron durante la hospitalización o se identificaron durante controles realizados en consulta externa. 


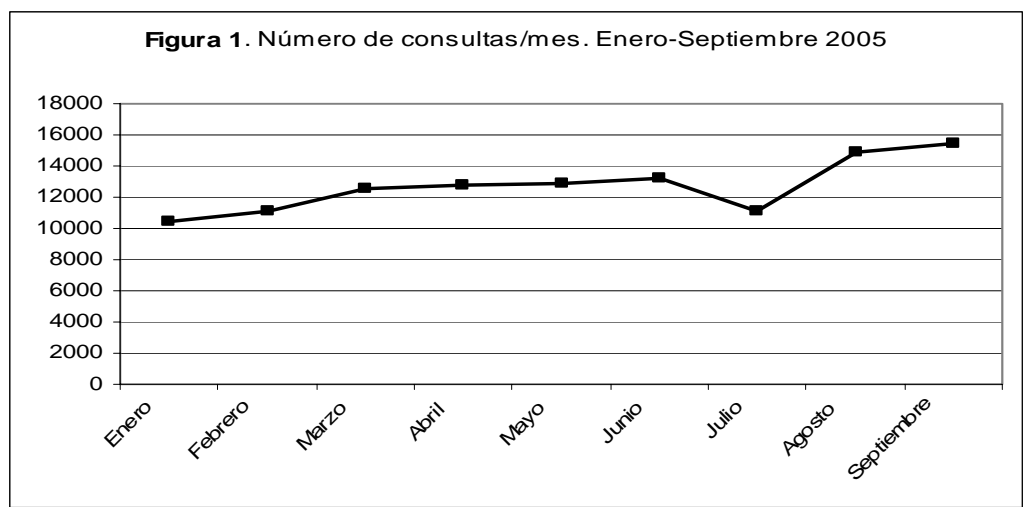

Las sospechas de RAM como motivo de consulta, provienen de los servicios de Consulta Externa (36,4 \%), Urgencias (18,2 \%) y Hospitalización Urgencias (4,5 \%). En Hospitalización Maternidad no se cuenta con reportes, aunque se presentó un caso de intoxicación intencional con Fenobarbital, esta no corresponde con la definición de RAM establecida en el presente documento.

Los reportes no clasificados como motivo de consulta se presentaron durante la hospitalización de maternidad (16 \%), hospitalización urgencias (2 $\%)$, controles (11,3\%) en pacientes que acuden especialmente al servicio de psiquiatría y administración de medicamentos durante la observación en urgencias $(2,2 \%)$.

Características demográficas de la población

El género femenino representa el $77 \%$ del total de pacientes que presentaron eventos adversos, esto debido a que en el CAMI presta el servicio de control prenatal. Excluyendo las pacientes de este servicio, el género femenino representa el $58 \%$.

El promedio de edad fue de 33,7 años (Desviación Estándar 21,1 años). Se presentó una mayor proporción de eventos en adultos jóvenes, niños y adolescentes (50\%). Por las características de los servicios prestados, el 44,2 \% de la población que presentó eventos adversos es población en edad fértil (19-36 años). Figura 2. 


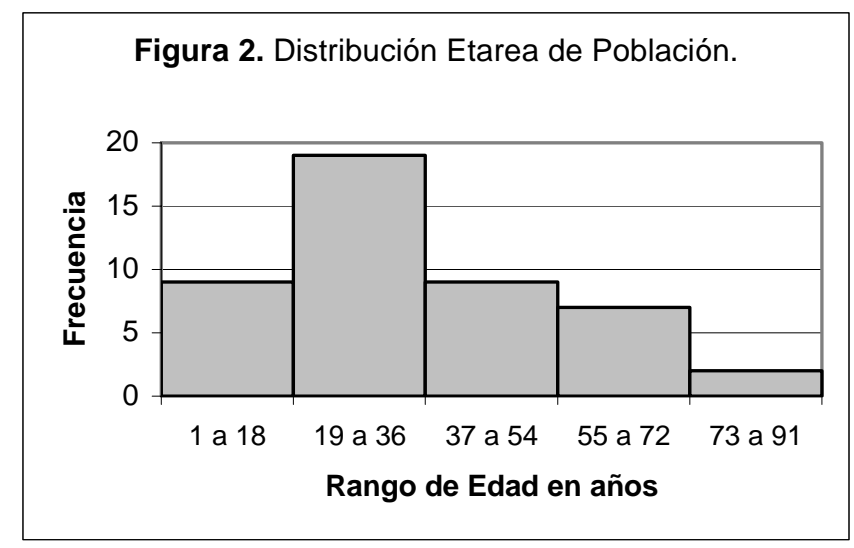

Clasificación de Reportes por Causalidad

El $13 \%$ de las RAMs reportadas fueron confirmadas; el $46 \%$ posibles, el 17 $\%$ probables y el $7 \%$ no clasificadas por deficiencia en la información de la historia clínica o el reporte y a dificultades en el seguimiento a pacientes, en especial cuando la severidad del evento ocasionó remisión a un centro de atención de mayor complejidad.

Clasificación de reportes por severidad

El $74 \%$ de los reportes no se relacionan con problemas que pongan en riesgo la vida del paciente o que prolonguen la hospitalización o incapaciten permanentemente, el $26 \%$ restante presentan reacciones que requieren de mayor atención dada su severidad. Durante el periodo de estudio una paciente falleció posterior a la administración de N-Butil Bromuro de Hioscina. Al momento de la redacción del presente documento, el caso se encontraba en estudio en el INVIMA.

\section{Clasificación órgano sistema afectado}

Los reportes recibidos se clasificaron en 7 grupos definidos como órgano sistema de mayor a menor porcentaje: Gastrointestinal (30,4 \%), Cardiovascular (17,4 \%), Sistema Nervioso Central (13\%), Piel (13\%), GinecoObstétrico (10,9\%); Otros (10,9\%) que incluye eventos como hipoglucemia, defectos visuales, entre otros y un reporte Genitourinario (4,3\%).

Medicamentos sospechosos 
Los antipsicóticos y antidepresivos representaron el 30,4 \% de los reportes, los suplementos nutricionales el 17,4 \%, los antibióticos, antiparasitarios y biológicos el 10,9\%, los analgésicos, antipiréticos y antiespasmódicos el 10,9 \%, los oxitócicos el 10,9 \%, los antiulcerosos el 8,7 \%; los anticonceptivos orales el 4,3 \%; los antidiarreicos el 2,2 \%; los broncodilatadores el 2,2 $\%$ y los hipoglicemiantes orales el 2,2 \%.

De manera concomitante en la mayoría de los casos los pacientes estaban recibiendo otros medicamentos como analgésicos, antibióticos, antidepresivos, antipsicóticos, antiulcerosos, anticoagulantes, antihipertensivos, corticoides, hipoglicemiantes y broncodilatadores.

\section{DISCUSIÓN}

Como en todos los programas de fármacovigilancia, los resultados tienen la limitación de representar solamente aquellos eventos que los médicos consideran importantes de acuerdo a su experiencia clínica y a la motivación que se les brinde. Para el caso de los medicamentos psiquiátricos, este especialista mostró mucho interés en el programa, sumado al hecho que el CAMI presenta un número elevado de consultas para esta especialidad. Dentro de los EA observados se encontró que los medicamentos empleados en trastornos psiquiátricos se encuentran relacionados con problemas de tipo hematológico, de los cuales se han reportado casos aislados que merecen especial atención $(7,8)$. Entre los trastornos hematológicos los más frecuentemente descritos son leucopenia, trombocitopenia y pancitopenia. Estos últimos pueden considerarse de gravedad y merecen atención por parte de los profesionales de la salud. Las reacciones adversas más frecuentemente asociadas con la terapia antipsicótica son los efectos extrapiramidales, pudiendo aparecer en más del $60 \%$ de los pacientes e incluyen: distonías (2-63\%), acatisias (10-20 \%) y efectos parkinsonianos (temblor, rigidez y bradikinesia). (1, 9).

Mención especial merece la oxitocina de la cual se presentaron reportes de fallo terapéutico considerado serio (hemorragia posparto), dado que fue necesario remitir a las pacientes a centros de mayor complejidad para su manejo. En un estudio realizado en urgencias por la universidad de Pisa (Italia), menciona que los Fallos Terapéuticos (FT) "son considerados responsables de un incremento significativo en la duración de la enfermedad del paciente, duración de la hospitalización y periodos de ausencia laboral, resultado de lo anterior se producen repercusiones en la disminución de la calidad de vida e incremento en el costo social" (10). Dentro de los factores que influyen en el fallo terapéutico están la inapropiada prescripción, pobre ad- 
herencia, factores farmacológicos (interacciones medicamentosas, tolerancia, etc), así como factores farmacéuticos (condiciones inapropiadas de almacenamiento, falsificaciones, administración inadecuada, etc) (11). El fallo terapéutico debe reportarse con especial atención para que no se convierta en argumento para una competencia desleal, descartando razones de uso del medicamento, farmacocinéticas y/o farmacodinámicas que permitan establecer una causa potencial de la falta de respuesta. Si se sospecha de un problema de calidad, se debe sustentar con argumentos técnicos y científicos, pues el reporte indiscriminado y mal intencionado no solo congestiona las actividades de los centros de fármacovigilancia sino que desvía su atención, dificultando la identificación de potenciales señales.

Los adultos mayores (55-91 años) considerados en otros estudios (12) como población en mayor riesgo de experimentar RAM, en este estudio no se registraron. Es posible que los médicos omitan información de éstos pacientes al considerar que son producto de la patología de base o que por llevar tiempo prolongado consumiendo medicamentos aceptan estos efectos indeseables como parte de la sintomatología de su enfermedad, pasando desapercibidos para ellos y para el personal de salud que atiende sus consultas. De ahí la importancia de orientar a los pacientes hacia la identificación de PRM.

Los resultados obtenidos durante el periodo de estudio sugieren al género femenino como grupo de riesgo considerándose de especial interés por las consecuencias que podría traer el uso inadecuado de medicamentos sobre el feto y las futuras madres. Así mismo se identificaron a los niños, adolescentes y adultos jóvenes como grupos en riesgo de presentar EA. Un alto porcentaje de los medicamentos sospechosos fueron administrados por vía oral. Las RAM que provienen de hospitalización cuenta con un número importante de medicamentos administrados por vía IV como es de esperarse para este servicio. El presente estudió es una contribución importante al programa nacional de fármacovigilancia que lidera el INVIMA y contribuyó a identificar a las gestantes como grupo de riesgo.

Agradecimientos. A los profesionales que colaboraron con el reporte de sospecha de RAM, asi como también a todas aquellas personas que lograron el contacto con el personal que labora en el hospital de Suba y suministraron datos de estadísticas de consulta en el CAMI Suba. 


\section{REFERENCIAS}

1. Schneeweiss S, Göttler M, Hasford J, Swoboda W, Hippius M, Hoffmann A, Et al. First results from intensified monitoring system to estimate drug related hospital admissions. Blackwell Science Ltd Br J Clin Pharmacol. 2001; 52:196-200.

2. World Health Organization. SIGNAL, Analyses of Adverse Reaction Reports in the WHO Database. Marzo 2003.

3. Segura O, Maldonado C E. Las reacciones adversas a medicamentos: una aproximación desde el punto de vista económico. Biomédica 2003; 23:401-7.

4. Tribiño G, Maldonado C, Segura O, Díaz J. Costos directos y aspectos clínicos de las reacciones adversas a medicamentos en pacientes hospitalizados en el servicio de medicina interna de una institución de tercer nivel de Bogotá. Biomédica 2006; 26:31-41.

5. Asociación Colombiana para el avance de la Investigación Clínica, Avanzar. Manual de Fármacovigilancia. Bogotá, Colombia; 2002.

6. Consenso de Granada sobre Problemas Relacionados con Medicamentos. Pharm Care. España. 1999; 1:107-112.

7. Garzón S, Maiquez V, Gutiérrez R, Rubio V, Gómez T, Calvo M, Campo R, Et al. Carbamazepina como posible causa de Diátesis Hemorrágica. Farmacia Hospitalaria; 1998; 22(6):313-315.

8. Calvo J, García F, Hernández J, Duarte J, Fisac P. Calmuntia Asencio M J. Anemia Aplasica y Carbamazepina. Descripción de un caso. Farmacia Hospitalaria 1998; 22(1): 46-48.

9. San Miguel M, Vila M, Díaz M, Azorín M, Fernández V, Robles S, Incidencia de efectos extrapiramidales en pacientes con Esquizofrenia tratados con Haloperidol solo o asociado a Biperideno. Farmacia Hospitalaria 1995; 19(4): 225-228.

10. Franceschi A, Tuccori M, Bocci G, Vannozzi F, Di Paolo A, Bárbara C, et al, Drug Therapeutic Failures in Emergency Departament Patiens. A University hospital experience. Pharmacological Research. 2004; 49:85-91.

11. Hartigan-Go KY, Wong JQ, Inclusion of therapeutic failures in adverse drug reactions. In: Aronson J, editor. Side effects of drugs. Annual 23. A worldwide yearly survey of new data and trends in adverse drug reaction. Amsterdam: Elsevier; 2000. pp. 27-33.

12. Gurwitz J, Field T, Harrold L, Rothschild J, Debellis K, Seger A, et al. Incidence and Preventability of Adverse Drug Events Among Older Persons in the Ambulatory Setting. American Medical Association. JAMA; 2003; 289(9): 1107 - 1116. 